\title{
新型不对称酞菁锌的合成和表征
}

\author{
俞孝伟 $a, b$ 詹传郎 ${ }^{b}$ 黄 彦*,a \\ ( ${ }^{a}$ 南京工业大学化学化工学院 材料化学工程国家重点实验室＼cjkstart南京 210009) \\ $\left({ }^{b}\right.$ 中国科学院化学研究所 北京分子科学国家实验室 中国科学院光化学重点实验室 北京 100190)
}

摘要 以 4-硝基邻苯二甲腈为主要原料, 合成了 2(3),9(10),16(17),23(24)-四-(正庚酰胺基)-酞菁锌、2(3),9(10),16(17), 23(24)-四-(甲氧基)-酞菁锌、2(3),9(10),16(17),23(24)-四-(对叔丁基苯氧基)-酞菁锌和 2(3),9(10),16(17),23(24)-四-(苯氧 基)-酞菁锌等 4 种对称型酞菁. 在此基础上, 选取 4-对叔丁基苯氧基邻苯二甲腈为前驱体, 采用概率法合成了含有氨 基、羧基的 2 种不对称唒菁锌 $\left(\mathrm{A}_{3} \mathrm{~B}\right)$.

关键词 锌酞菁; 不对称唒菁; 合成; 前驱体; 概率法

\section{Synthesis and Characterization of New Asymmetrical Phthalocyanine Zinc}

\author{
Yu, Xiaowei ${ }^{a, b} \quad$ Zhan, Chuanlang $^{b} \quad$ Huang, Yan*,a \\ ( ${ }^{a}$ State Key Laboratory of Materials-Oriented Chemical Engineering, College of Chemistry and Chemical Engineering, \\ Nanjing University of Technology, Nanjing 210009) \\ ( ${ }^{b}$ Beijing National Laboratory for Molecular Sciences (BNLMS), Laboratory of Photochemistry, Institute of Chemistry, \\ Chinese Academy of Sciences, Beijing 100190)
}

\begin{abstract}
Four kinds of symmetrical phthalocyanine zincs (PCZn) 2(3),9(10),16(17),23(24)-(tetraheptanylcarbonyl amino)-phthalocyanine zinc, 2(3),9(10),16(17),23(24)-tetramethoxy-phthalocyanine zinc, 2(3),9(10),16(17),23(24)-tetra-(4tert-butylphenoxyl)-phthalocyanine zinc, and 2(3),9(10),16(17),23(24)-tetraphenoxyl-phthalocyanine zinc were synthesized from 4-nitro-phthalonitrile. Moreover, the 4-(4-tert-butylphenoxyl)phthalonitrile was employed as a precursor in the synthesis of two kinds of asymmetrical PCZn $\left(\mathrm{A}_{3} \mathrm{~B}\right)$ containing either amino- or carboxyl-group by probability method.

Keywords phthalocyanine zinc; asymmetrical phthalocyanine; synthesis; precursor; probability method
\end{abstract}

从最初的染料、颜料到如今的医学、光化学等领域, 酞菁以其独特的化学稳定性和优异的光学特征, 自发现 以来一直受到人们的关注. 但是, 酞菁系列化合物有着 较差的溶解性和成膜性, 在应用中受到很大限制. 为此, 人们研发了多种酞菁衍生物 ${ }^{[1,2]}$, 其中以对称性酞菁 $\left(\mathrm{A}_{4}\right)$ 和 $1: 3$ 不对称酞菁 $\left(\mathrm{A}_{3} \mathrm{~B}\right)$ 为主. 由于不对称酞菁在合成 和分离提纯方面存在诸多问题, 因而成为酞菁衍生物合 成中的难点. 本工作以 4-硝基邻苯二甲腈为主要原料, 合成了 2(3),9(10),16(17),23(24)-四-(正庚酰胺基)-酞菁 锌、2(3),9(10),16(17),23(24)-四-(甲氧基)-酞菁锌、 2(3),9(10),16(17),23(24)-四-(对叔丁基苯氧基)-酞菁锌 和2(3),9(10), 16(17),23(24)-四-(苯氧基)-酞菁锌等4种对
称型酞菁. 在此基础上, 选取 4-对叔丁基苯氧基邻苯二 甲腈为前驱体，通过概率法合成了含有氨基、羧基的 2 种不对称酞菁锌 $\left(\mathrm{A}_{3} \mathrm{~B}\right)$, 并采用柱层析分离对它们进行 了提纯.

\section{1 实验部分}

\section{1 实验仪器及药品}

Bruker 300, 400 或 $600 \mathrm{~Hz}$ 核磁共振仪(内标为 TMS, 溶剂为 $\mathrm{CDCl}_{3}$ 或 DMSO- $d_{6}$ ); Bruker Biflex III 飞行时间 质谱仪(MALTI-TOF)或(ESI-MS); Millipore-Q 纯水机; AP 250D 分析天平. 所用化学试剂均为分析纯. 柱层析 硅胶产自青岛海洋化工厂(200 300 目).

\footnotetext{
* E-mail: huangy@njut.edu.cn

Received September 29, 2011; revised November 25, 2011; published online December 23, 2011

Project supported by the National Natural Science Foundation of China (No. 20973182).

国家自然科学基金(No. 20973182)资助项目.
} 


\section{2 合成}

\subsection{1 前躯体的合成}

合成路线如 Scheme 1 所示. 具体合成方法与表征 结果如下:

4-氨基邻苯二甲腈 $(2)^{[3]}$ : 取 4-硝基邻苯二甲腈(1) $3.0 \mathrm{~g}$ 溶于 $50 \mathrm{~mL}$ 甲醇, 加入浓盐酸 $15 \mathrm{~mL}$, 加热回流, 将铁粉 $4 \mathrm{~g}$ 每隔 $15 \mathrm{~min}$ 分四次加入, 反应 $5 \mathrm{~h}$, 过滤; 滤 液用旋转蒸发仪旋干, 以二氯甲烷和乙酸乙酯 $(V: V=$ $1: 1)$ 为流动相进行柱层析提纯, 取第二个点, 得到白 色固体 $21.55 \mathrm{~g}$, 产率 $62.5 \%$. ${ }^{1} \mathrm{H}$ NMR $\left(\mathrm{CDCl}_{3}, 400\right.$ MHz) $\delta$ : $7.53(\mathrm{~d}, J=8.0 \mathrm{~Hz}, 1 \mathrm{H}), 6.95(\mathrm{~s}, 1 \mathrm{H}), 6.86$ (d, $J=$ $8.0 \mathrm{~Hz}, 1 \mathrm{H}), 4.43(\mathrm{~s}, 2 \mathrm{H}) ;{ }^{13} \mathrm{C} \mathrm{NMR}\left(\mathrm{CDCl}_{3}, 100 \mathrm{MHz}\right) \delta$ : 151.5, 135.1, 128.2, 118.3, 117.8, 120.1, 106.0; EI-MS m/z (\%): 143 (100), $116(30)$.

4-正庚酰胺基邻苯二甲腈 $(3)^{[4]}$ : 取 4-氨基邻苯二腈 (2) $1.0 \mathrm{~g}$ 溶于二氯甲烷, 冰浴, 慢慢加入庚酰氯 $2 \mathrm{~mL}$, DIEPA $2 \mathrm{~mL}$, 温度升至室温后继续反应 $12 \mathrm{~h}$, 用水 $/ 二$ 氯甲烷萃取; 取有机相并进行旋干, 再用二氯甲烷、石 油醚重结晶 3 次, 得到白色固体产物 $31.4 \mathrm{~g}$, 产率 74.4\%. ${ }^{1} \mathrm{H}$ NMR $\left(\mathrm{CDCl}_{3}, 400 \mathrm{MHz}\right) \delta: 8.15(\mathrm{~s}, 1 \mathrm{H}), 7.86$ (d, $J=8.0 \mathrm{~Hz}, 1 \mathrm{H}), 7.75(\mathrm{~d}, J=8.0 \mathrm{~Hz}, 1 \mathrm{H}), 7.45(\mathrm{~s}, 1 \mathrm{H})$, $2.45(\mathrm{t}, J=4.0,8.0 \mathrm{~Hz}, 2 \mathrm{H}), 1.76(\mathrm{t}, J=8.0,8.0 \mathrm{~Hz}, 2 \mathrm{H})$, $1.35(\mathrm{~s}, 2 \mathrm{H}), 1.30(\mathrm{~s}, 6 \mathrm{H}), 0.89(\mathrm{~d}, J=8.0 \mathrm{~Hz}, 3 \mathrm{H}) ;{ }^{13} \mathrm{C}$ NMR $\left(\mathrm{CDCl}_{3}, 100 \mathrm{MHz}\right) \delta: 173.0,143.3,134.5,123.6$, 123.1, 116.7, 116.0, 115.7, 109.2, 37.7, 31.7, 29.2, 29.1, 25.3, 22.6, 14.1; EI-MS m/z (\%): 269 (30), 203 (100), 160 (50), 133 (25), 127 (35).

4-甲氧基邻苯二甲腈 $(4)^{[5,6]}$ : 将 4-硝基邻苯二腈(1) $3.0 \mathrm{~g}$ 溶解在 $50 \mathrm{~mL}$ 的 DMSO 中, 加入甲醇 $6 \mathrm{~mL}$, $\mathrm{LiOH} \cdot 5 \mathrm{H}_{2} \mathrm{O} 1.5 \mathrm{~g}$, 在氮气保护下于 $50{ }^{\circ} \mathrm{C}$ 反应 $12 \mathrm{~h}$, 冷 却至室温; 将反应液倒入饱和 $\mathrm{NaCl}$ 水溶液中, 抽滤析 出的沉淀, 并用饱和 $\mathrm{NaCl}$ 水溶液洗涤; 真空干燥后, 用 二氯甲烷和甲醇 $(V: V=10: 1 \sim 2: 1$ 梯度)柱层析分离, 取第二个点, 得到褐色固体产物 $41.2 \mathrm{~g}$, 产率 $43.8 \%$. ${ }^{1} \mathrm{H}$
NMR (DMSO- $\left.d_{6}, 400 \mathrm{MHz}\right) \delta: 7.68(\mathrm{~d}, J=8.0 \mathrm{~Hz}, 1 \mathrm{H})$, $7.25(\mathrm{~s}, 1 \mathrm{H}), 6.84(\mathrm{~d}, J=8.0 \mathrm{~Hz}, 1 \mathrm{H}), 3.36(\mathrm{~s}, 3 \mathrm{H}) ;{ }^{13} \mathrm{C}$ NMR (DMSO- $\left.d_{6}, 100 \mathrm{MHz}\right) \delta: 166.8,135.2,128.5,128.0$, 121.3, 117.9, 113.9, 113.6, 50.8; ESI-MS $m / z$ (\%): 158 (M $\left.-\mathrm{H}^{+}, 100\right)$.

4-对叔丁基苯氧基邻苯二甲腈 $(5)^{[7]}$ : 将 $5.0 \mathrm{~g}$ 对叔丁 基苯酚和 4-硝基邻苯二腈(1) $3.0 \mathrm{~g}$ 溶解在 $100 \mathrm{~mL}$ DMSO 中, 加入 $2.0 \mathrm{~g} \mathrm{LiOH} \cdot 5 \mathrm{H}_{2} \mathrm{O}$, 在氮气保护下于 50 ${ }^{\circ} \mathrm{C}$ 反应 $12 \mathrm{~h}$, 冷却至室温; 将反应液倒入饱和 $\mathrm{NaCl}$ 水溶 液中, 析出黄色沉淀, 抽滤并用饱和 $\mathrm{NaCl}$ 水溶液洗涤; 真空干燥后, 用二氯甲烷为流动相进行柱层析提纯, 取 第一个点. 得到淡绿色固体产物(5) $2.1 \mathrm{~g}$, 产率 43.9\%. ${ }^{1} \mathrm{H}$ NMR $\left(\mathrm{CDCl}_{3}, 400 \mathrm{MHz}\right) \delta: 7.73(\mathrm{~d}, J=8.0 \mathrm{~Hz}, 1 \mathrm{H})$, 7.48 (d, $J=8.0 \mathrm{~Hz}, 2 \mathrm{H}), 7.24$ (s, 2H), 7.01 (d, $J=8.0 \mathrm{~Hz}$, $2 \mathrm{H}), 1.36$ (s, 9H); ${ }^{13} \mathrm{C} \mathrm{NMR}\left(\mathrm{CDCl}_{3}, 100 \mathrm{MHz}\right) \delta: 161.2$, $154.9,143.2,136.2,127.2,125.8,122.3,121.6,116.6$, 115.8, 107.9, 40.1, 39.3, 39.1, 38.9; EI-MS $m / z$ (\%): 276 $\left(\mathrm{M}-\mathrm{H}^{+}, 100 \%\right)$.

4-苯氧基邻苯二甲腈 $(6)^{[8]}$ : 将 $4.0 \mathrm{~g}$ 苯酚和 $3.0 \mathrm{~g} \mathrm{4-}$ 硝基邻苯二腈(1)溶解在 $100 \mathrm{~mL}$ DMSO 中, 加 $2.0 \mathrm{~g}$ $\mathrm{LiOH} \cdot 5 \mathrm{H}_{2} \mathrm{O}$, 在氮气保护下于 $50{ }^{\circ} \mathrm{C}$ 反应 $12 \mathrm{~h}$, 冷却至 室温; 将反应液倒入饱和 $\mathrm{NaCl}$ 水溶液中, 析出黄色沉 淀, 抽滤并用饱和 $\mathrm{NaCl}$ 水溶液洗涤; 真空干燥后, 用二 氯甲烷/石油醚 $(V: V=3: 1)$ 为流动相进行柱层析提纯, 取第一个点，得到黄色固体产物 $61.9 \mathrm{~g}$, 产率 $49.8 \% .{ }^{1} \mathrm{H}$ NMR (DMSO- $\left.d_{6}, 400 \mathrm{MHz}\right) \delta: 8.11(\mathrm{~d}, J=8.0 \mathrm{~Hz}, 1 \mathrm{H})$, $7.78(\mathrm{~s}, 1 \mathrm{H}), 7.51(\mathrm{t}, J=8.0,8.0 \mathrm{~Hz}, 2 \mathrm{H}), 7.32-7.38(\mathrm{~m}$, $2 \mathrm{H}), 7.20(\mathrm{~d}, J=8.0 \mathrm{~Hz}, 2 \mathrm{H}) ;{ }^{13} \mathrm{C} \mathrm{NMR}\left(\mathrm{CDCl}_{3}, 100 \mathrm{MHz}\right)$ $\delta: 161.3,161.0,148.8,136.3,135.7,130.66,125.8,125.4$, 122.7, 121.9, 120.3, 117.0, 116.7, 108.1; EI-MS $m / z(\%)$ : 276 (15), 261 (100), 233 (10); EI-MS $m / z$ (\%): 220 (100), 192 (25), 77 (20).

\subsection{2 对称型酞菁的合成}

合成路线如 Scheme 2 所示. 具体合成方法与表征 结果如下:

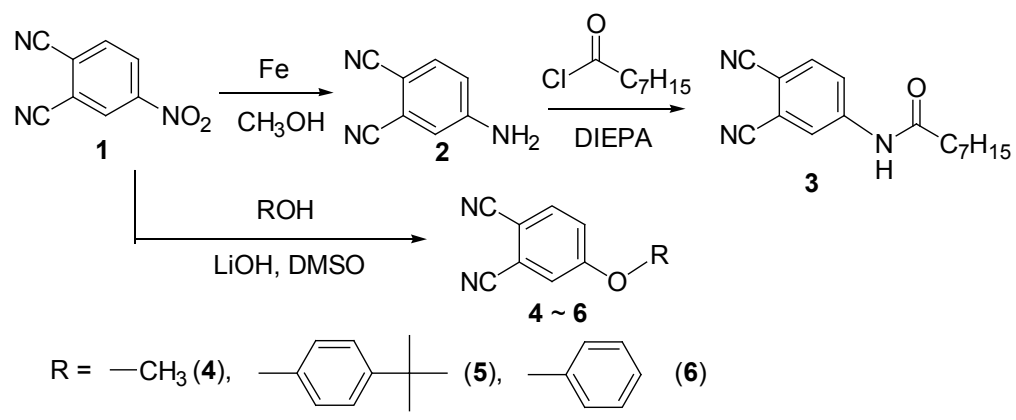

Scheme 1 


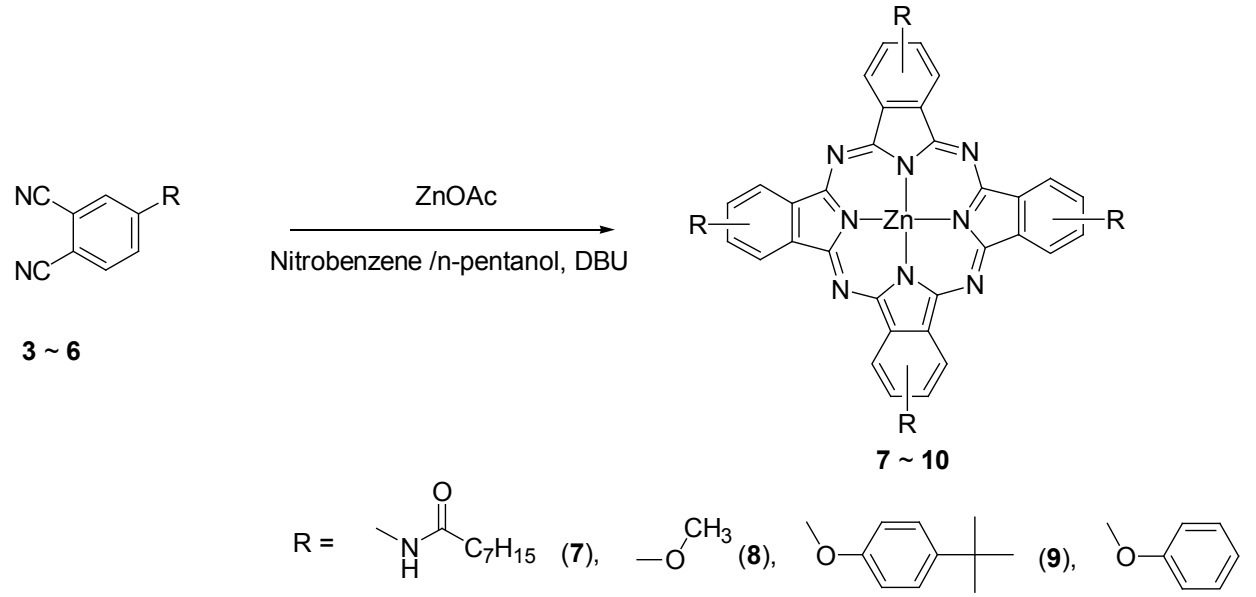

(10)

\section{Scheme 2}

2(3),9(10),16(17),23(24)-四-(正庚酰胺基)-酞菁锌 $(7)^{[9,10]}$ : 称取 $1.0 \mathrm{~g} \mathrm{3}, 0.2 \mathrm{~g}$ 醋酸锌, $1.0 \mathrm{~g}$ 尿素, $0.013 \mathrm{~g}$ 钿酸铵溶于 $30 \mathrm{~mL}$ 硝基苯中, 在 $180{ }^{\circ} \mathrm{C}$ 下反应 $5 \mathrm{~h}$, 冷 却; 将甲醇加入到反应液中则有沉淀析出; 经抽滤、水 洗、抽干后, 用 $\mathrm{THF} / \mathrm{H}_{2} \mathrm{O}$ 进行重结晶, 得到蓝绿固体产 物 $70.9 \mathrm{~g}$, 产率 85.0\%. ${ }^{1} \mathrm{H}$ NMR (DMSO- $\left.d_{6}, 400 \mathrm{MHz}\right) \delta$ : $10.66(\mathrm{~s}, 4 \mathrm{H}), 9.65 \sim 9.89(\mathrm{~m}, 4 \mathrm{H}), 8.98 \sim 9.20(\mathrm{~m}, 4 \mathrm{H})$, $8.16 \sim 8.51(\mathrm{~m}, 4 \mathrm{H}), 2.58(\mathrm{~s}, 8 \mathrm{H}), 1.80(\mathrm{~s}, 24 \mathrm{H}), 1.35(\mathrm{~s}$, $8 \mathrm{H}), 0.91\left(\mathrm{~d}, J=8.0 \mathrm{~Hz}, 12 \mathrm{H}\right.$ ); ${ }^{13} \mathrm{C}$ NMR (DMSO- $d_{6}, 100$ MHz) $\delta: 167.4,158.7,158.5,155.3,155.2,155.0,154.9$, 152.2, 116.7, 116.0, 115.7, 109.2, 37.7, 31.7, 29.2, 29.1, 25.3, 22.6, 14.1; TOF-MS m/z (\%): 1139.5 (100). Anal. calcd for $\mathrm{C}_{64} \mathrm{H}_{76} \mathrm{~N}_{12} \mathrm{O}_{4} \mathrm{Zn} \cdot \mathrm{H}_{2} \mathrm{O}$ : C 66.20, H 8.33, N 14.48; found C 66.10, H 6.91, N 14.21.

2(3),9(10),16(17),23(24)- 四一(甲氧基 )- 酞菁锌

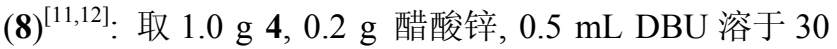
$\mathrm{mL}$ 正戊醇中, 在 $135{ }^{\circ} \mathrm{C}$ 下反应 $5 \mathrm{~h}$, 冷却; 将甲醇加入 到反应液中, 沉淀析出, 经抽滤、水洗、抽干后备用. 用 $\mathrm{THF} / \mathrm{H}_{2} \mathrm{O}$ 进行重结晶得到墨绿色固体产物 $80.7 \mathrm{~g}$, 产率 $63.6 \%$. ${ }^{1} \mathrm{H}$ NMR (DMSO- $\left.d_{6}, 400 \mathrm{MHz}\right) \delta: 8.25 \sim 9.30(\mathrm{~m}$, $8 \mathrm{H}), 7.72 \sim 7.70(\mathrm{~m}, 4 \mathrm{H}), 3.36(\mathrm{~s}, 9 \mathrm{H}) ;{ }^{13} \mathrm{C} \mathrm{NMR}\left(\mathrm{CDCl}_{3}\right.$, $100 \mathrm{MHz}) \delta$ : $167.4,158.7,158.5,155.3,155.2,155.0$, 154.9, 152.2, 151.3, 150.2, 50.8; TOF-MS $m / z$ (\%): 696.1 (100). Anal. calcd for $\mathrm{C}_{36} \mathrm{H}_{24} \mathrm{~N}_{8} \mathrm{O}_{4} \mathrm{Zn}: \mathrm{C} 61.94, \mathrm{H} 3.47, \mathrm{~N}$ 16.05; found C 61.81, H 3.51, N 15.65 .

2(3),9(10),16(17),23(24)-四-(对叔丁基苯氧基)-酞

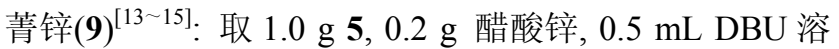
于 $30 \mathrm{~mL}$ 正戊醇中, 在 $135{ }^{\circ} \mathrm{C}$ 下反应 $5 \mathrm{~h}$, 冷却; 将甲醇 加入到反应液中则有沉淀析出, 经抽滤、水洗、抽干后 备用. 用二氯甲烷/乙酸乙酯 $(V: V=1: 1)$ 进行柱层析, 得到蓝色固体产物 $90.8 \mathrm{~g}$, 产率 $66.2 \%$. ${ }^{1} \mathrm{H}$ NMR
(DMSO- $\left.d_{6}, 400 \mathrm{MHz}\right) \delta: 8.25 \sim 9.30(\mathrm{~m}, 8 \mathrm{H}), 7.72 \sim 7.81$ $(\mathrm{m}, 4 \mathrm{H}), 7.57 \sim 7.70(\mathrm{~m}, 8 \mathrm{H}), 7.30 \sim 7.40(\mathrm{~m}, 8 \mathrm{H}), 1.38(\mathrm{~d}$, $J=12 \mathrm{~Hz}, 36 \mathrm{H}$ ); ${ }^{13} \mathrm{C}$ NMR (DMSO- $d_{6}, 100 \mathrm{MHz}$ ) $\delta: 167.4$, $158.7,158.5,155.3,155.2,155.0,154.9,152.2,151.3$, $150.2,146.9,141.4,139.7,133.1,132.2,132.0,129.1$, $127.5,127.0,125.4,124.0,119.5,116.4,110.6,106.9$, 32.6, 31.9, 30.9, 30.5, 29.5; TOF-MS $m / z$ (\%): 1168.4 (100). Anal. calcd for $\mathrm{C}_{72} \mathrm{H}_{64} \mathrm{~N}_{8} \mathrm{O}_{4} \mathrm{Zn} \cdot 2 \mathrm{H}_{2} \mathrm{O}$ : C $71.77 \mathrm{H}$ 5.69, N 9.30; found C 71.87, H 5.70, N 8.99.

2(3),9(10),16(17),23(24)- 四-(苯氧基 )- 酞菁锌

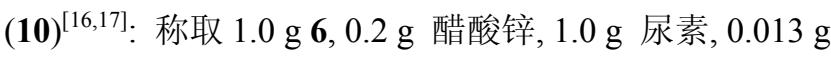
钿酸铵溶于 $30 \mathrm{~mL}$ 硝基苯中, 在 $180{ }^{\circ} \mathrm{C}$ 下反应 $5 \mathrm{~h}$, 冷 却; 将甲醇加入到反应液中则有沉淀析出; 经抽滤、水 洗、抽干后. 用二氯甲烷/乙酸乙酯 $(V: V=3: 1)$ 进行柱 层析提纯得到蓝色固体产物 $\mathbf{1 0} 0.9 \mathrm{~g}$, 产率 $65.2 \% .{ }^{1} \mathrm{H}$ NMR (DMSO- $\left.d_{6}, 400 \mathrm{MHz}\right) \delta: 8.25 \sim 9.30(\mathrm{~m}, 8 \mathrm{H})$, $7.72 \sim 7.81(\mathrm{~m}, 4 \mathrm{H}), 7.57 \sim 7.70(\mathrm{~m}, 8 \mathrm{H}), 7.30 \sim 7.40(\mathrm{~m}$, $12 \mathrm{H}$ ); ${ }^{13} \mathrm{C}$ NMR (DMSO- $\left.d_{6}, 100 \mathrm{MHz}\right) \delta: 158.4,158.3$, $156.8,151.4,151.2,151.0,139.1,139.0,132.4,130.5$, 130.4, 124.3, 123.4, 119.8, 110.2; TOF-MS $m / z$ (\%): 944.1 (100). Anal. calcd for $\mathrm{C}_{56} \mathrm{H}_{32} \mathrm{~N}_{8} \mathrm{O}_{4} \mathrm{Zn} \cdot 2 \mathrm{H}_{2} \mathrm{O}$ : C 68.6, H 3.7, $\mathrm{N}$ 11.43; found C 69.01, H 3.62, N 11.63.

\subsection{3 不对称酞菁的合成}

合成路线如 Scheme 3 所示. 具体合成方法与表征 结果如下:

2(3),9(10),16(17)-三-(对叔丁基苯氧基)-23(24)-(氨 基)-酞菁锌(12) ${ }^{[18]}$ : 称取 $1.0 \mathrm{~g}(\mathbf{9}), 0.1 \mathrm{~g} \mathbf{1}$ (前两者物质的 量比 $5: 1), 0.3 \mathrm{~g}$ 醋酸锌, $1.0 \mathrm{~g}$ 尿素, $0.013 \mathrm{~g}$ 钿酸铵溶于 $30 \mathrm{~mL}$ 硝基苯中, 在 $180{ }^{\circ} \mathrm{C}$ 下反应 $5 \mathrm{~h}$, 冷却; 将甲醇 加入到反应液中则有沉淀析出, 经抽滤、水洗、抽干得 到含化合物 11 的混合物，将该混合物重新溶于 DMF 


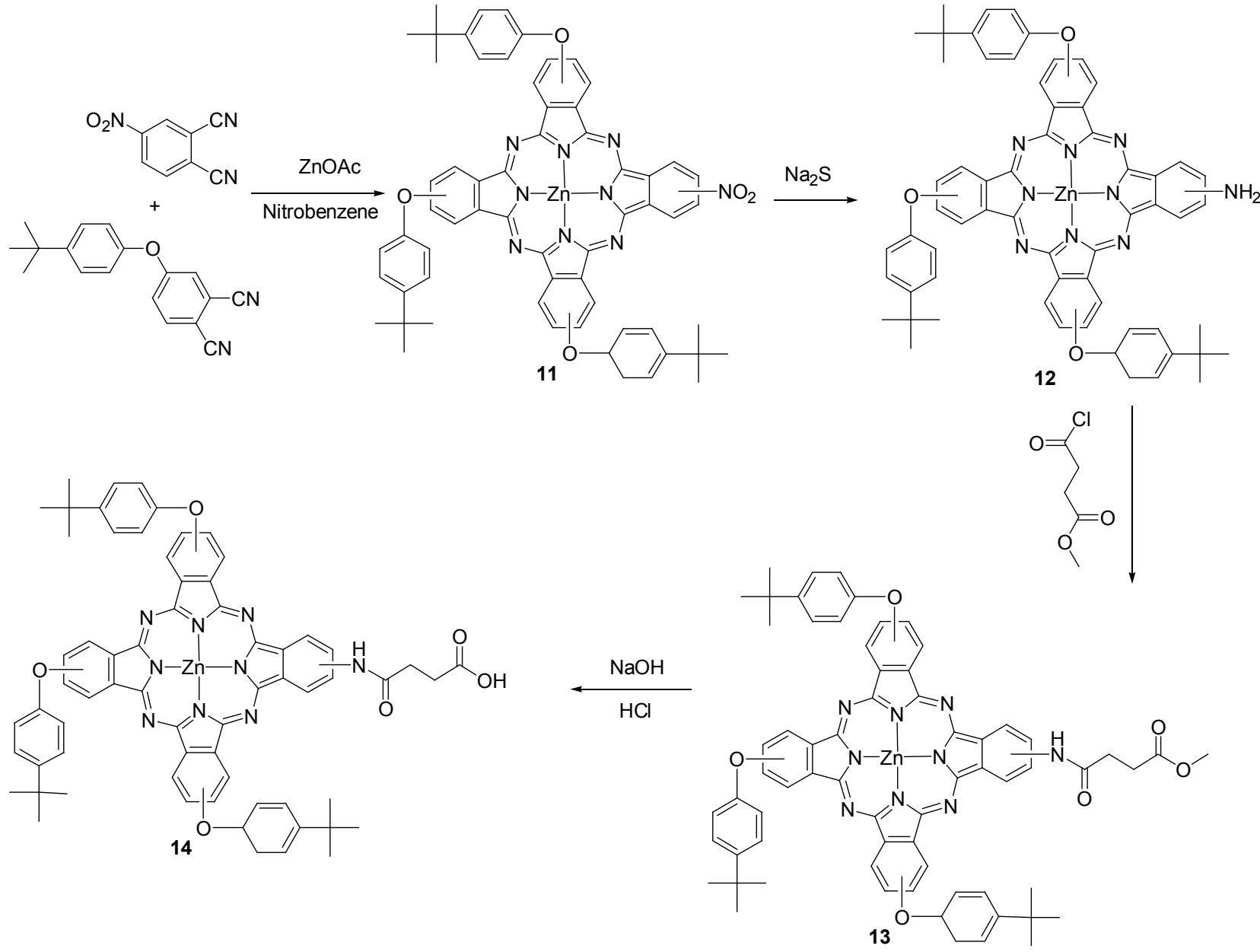

Scheme 3

并加入过量硫化钠, 在 $50{ }^{\circ} \mathrm{C}$ 下反应 $5 \mathrm{~h}$ 后冷却; 将甲醇 加入到反应液中则有沉淀析出, 经抽滤、水洗、抽干后, 用石油醚/乙酸乙酯 $(V: V=3: 1)$ 、乙酸乙酯进行柱层析 提纯, 第一个点为对称型酞菁产物 $\mathbf{9}$, 第二个点则为不 对称酞菁氨基取代物, 旋干即可得到草绿固体产物 $\mathbf{1 2}$ $0.2 \mathrm{~g}$, 产率 33.4\%. ${ }^{1} \mathrm{H}$ NMR (DMSO- $d_{6}, 400 \mathrm{MHz}$ ) $\delta$ : $8.25 \sim 9.30(\mathrm{~m}, 8 \mathrm{H}), 7.72 \sim 7.81(\mathrm{~m}, 4 \mathrm{H}), 7.57 \sim 7.70(\mathrm{~m}$, $6 \mathrm{H}), 7.30 \sim 7.40(\mathrm{~m}, 6 \mathrm{H}), 6.37(\mathrm{~s}, 2 \mathrm{H}), 1.38(\mathrm{~d}, J=12 \mathrm{~Hz}$, $27 \mathrm{H}$ ); ${ }^{13} \mathrm{C}$ NMR (DMSO- $\left.d_{6}, 100 \mathrm{MHz}\right) \delta: 167.4,158.7$, $158.5,155.3,155.2,155.0,154.9,152.2,151.3,150.2$, $146.9,141.4,139.7,133.1,132.2,132.0,129.1,127.5$, 127.0, 125.4, 124.0, 119.5, 116.4, 110.6, 106.9, 34.9, 34.7, 32.6, 31.9, 30.9, 30.5, 29.5; TOF-MS $m / z$ (\%): 1035.3 (100). Anal. calcd for $\mathrm{C}_{62} \mathrm{H}_{53} \mathrm{~N}_{9} \mathrm{O}_{3} \mathrm{Zn} \cdot 5 \mathrm{H}_{2} \mathrm{O}$ : C 66.04, $\mathrm{H}$ 5.63, N 11.18; found C 61.21, H 5.01, N 10.41.

2(3),9(10),16(17)-三-(对叔丁基苯氧基)-23(24)-(丁 二酸单乙酯酰胺基)-酞菁锌(13 $)^{[19,20]}$ : 称取 $0.2 \mathrm{~g} 12$ 溶于 $10 \mathrm{~mL}$ THF, 冰浴, 慢慢加入 $0.3 \mathrm{~mL}$ 丁二酸单酯酰氯和 $0.3 \mathrm{~mL}$ DIEPA, 温度升至室温后反应 $5 \mathrm{~h}$, 用乙酸乙酯/
二氯甲烷 $(V: V=3: 1)$ 为流动相, 进行柱分析提纯, 取 第二个点, 得到蓝绿固体产物 $130.18 \mathrm{~g}$, 产率 $81.0 \% .{ }^{1} \mathrm{H}$ NMR (DMSO- $\left.d_{6}, 400 \mathrm{MHz}\right) \delta: 8.25 \sim 9.30(\mathrm{~m}, 8 \mathrm{H})$, $7.72 \sim 7.81(\mathrm{~m}, 4 \mathrm{H}), 7.57 \sim 7.70(\mathrm{~m}, 6 \mathrm{H}), 7.30 \sim 7.40(\mathrm{~m}$, $6 \mathrm{H}), 6.8(\mathrm{~s}, 1 \mathrm{H}), 3.76(\mathrm{~d}, J=8 \mathrm{~Hz}, 3 \mathrm{H}), 3.02(\mathrm{~s}, 2 \mathrm{H}), 2.91$ (s, 2H), $1.38(\mathrm{~d}, J=12 \mathrm{~Hz}, 27 \mathrm{H}) ;{ }^{13} \mathrm{C}$ NMR (DMSO- $d_{6}$, $100 \mathrm{MHz}) \delta: 173.1,170.2,158.3,154.8,154.3,152.2$, $146.5,139.1,138.4,132.5,131.5,128.7,123.4,119.3$, $118.9,111.9,65.0,51.5,34.3,31.4,30.0,28.8,18.7$; TOF-MS $\mathrm{m} / \mathrm{z}$ (\%): 1135.3 (100). Anal. calcd for $\mathrm{C}_{67} \mathrm{H}_{61} \mathrm{~N}_{9} \mathrm{O}_{6} \mathrm{Zn} \cdot \mathrm{H}_{2} \mathrm{O}$ : C 68.80, H 5.26, N 10.72; found $\mathrm{C}$ 65.84, H 5.61, N 10.62 .

2(3),9(10),16(17)-三-(对叔丁基苯氧基)-23(24)-(丁 二酸单酸酰胺基)-酞菁锌(14) ${ }^{[21]}$ : 称取 $0.1 \mathrm{~g} 13$ 溶于 5 $\mathrm{mL}$ THF, 加入 $1 \mathrm{~mol} / \mathrm{L}$ 的 $\mathrm{NaOH}$ 溶液调 $\mathrm{pH}$ 值至 11, 常 温反应 $5 \mathrm{~h}$, 加入 $1 \mathrm{~mol} / \mathrm{L}$ 的盐酸调 $\mathrm{pH}$ 值至 6 ; 加入水和 二氯甲烷进行萃取, 取下层溶液旋干, 用乙酸乙酯/甲醇 $(V: V=3: 1)$ 为流动相, 进行柱层析提纯, 取第二个 点，旋干得到蓝绿固体产物 $140.09 \mathrm{~g}$, 产率 $91.2 \% .{ }^{1} \mathrm{H}$ 
NMR (DMSO- $\left.d_{6}, 400 \mathrm{MHz}\right) \delta: 10.8(\mathrm{~s}, 1 \mathrm{H}), 8.25 \sim 9.30$ $(\mathrm{m}, 8 \mathrm{H}), 7.72 \sim 7.81(\mathrm{~m}, 4 \mathrm{H}), 7.57 \sim 7.70(\mathrm{~m}, 6 \mathrm{H}), 7.30 \sim$ $7.40(\mathrm{~m}, 6 \mathrm{H}), 6.8(\mathrm{~s}, 1 \mathrm{H}), 2.76(\mathrm{~s}, 2 \mathrm{H}), 2.89(\mathrm{~s}, 2 \mathrm{H}), 1.38$ $(\mathrm{d}, J=12 \mathrm{~Hz}, 27 \mathrm{H}) ;{ }^{13} \mathrm{C}$ NMR (DMSO- $\left.d_{6}, 100 \mathrm{MHz}\right) \delta$ : $174.0,170.6,158.6,154.3,146.6,145.7,139.2$, 127.1, $124.8,123.7,119.2,34.3,31.4,30.4,29.2,29.0,28.8$; TOF-MS m/z (\%): 1135.3 (100). Anal. calcd for $\mathrm{C}_{66} \mathrm{H}_{59} \mathrm{~N}_{9} \mathrm{O}_{6} \mathrm{Zn} \cdot 2 \mathrm{H}_{2} \mathrm{O}: \mathrm{C}: 67.54, \mathrm{H}$ 5.24, N 10.74; found $\mathrm{C}$ 65.09, H 5.61, N 10.61 .

\section{2 结果与讨论}

\section{1 不对称酞菁前驱体的选择}

在对称型酞菁的合成过程中，我们发现正庚酰胺 基、甲氧基取代的对称型酞菁在二氯甲烷、乙酸乙酯等 常用溶剂中只能微溶, 而对苯氧基和叔丁基苯氧基取代 的对称型酞菁在二氯甲烷和乙酸乙酯溶剂中却能很好 地溶解. 这是由于苯氧基和对叔丁基苯氧基所具有的空 间位阻效应降低了酞菁的 $\pi-\pi$ 相互作用，阻碍了酞菁二 聚体甚至多聚体的形成，因而比相应的正庚酰胺基和甲 氧基取代的直链酞菁表现出更好的溶解性. 在不对称酞 菁的合成中, 溶解性的好坏直接影响着其后续分离和提 纯的难度. 对叔丁基苯氧基比苯氧基的空间位阻更大, 溶解度也更好，因此我们在不对称酞菁的合成中选取了 4-对叔丁基苯氧基邻苯二甲腈作为前驱体.

\section{2 合成路线及方法的选择}

\subsubsection{4-硝基邻苯二腈代替 4-氨基邻苯二腈}

若采用 4-氨基邻苯二腈作为前躯体, 其在酞菁的合 成过程中容易被取代，因而需要 Boc 保护，在合成酞菁 后必须再脱除 Boc. 这使反应步骤中多出引入 Boc 的引 入和脱除. 因此, 我们在本工作中选用 4-硝基邻苯二腈 作为合成酞菁的前驱体，合成后再将硝基还原.

\subsection{2 合成方法的选择}

本工作中以邻苯二甲腈衍生物为原料、金属盐为 “模板剂”来合成酞菁配合物，并分别采用 DBU 催化法、 硝基苯惰性溶剂法合成对称型酞菁，这两种方法较其它 方法条件温和，其中 DBU 催化法是目前合成酞菁的主 流方法, 有着反应温度低、毒性小、产率高等特点. 但 在合成含硝基的不对称酞菁时，我们采用了硝基苯惰性 溶剂法, 这是为了防止苯环上的硝基在 DUB 强碱条件 下被反应溶剂正戊醇所取代.

\section{3 分离}

不对称酞菁的分离提纯, 一直是其合成过程中的难 点. 在本工作中, 我们绕开含硝基不对称酞菁的分离, 而是将硝基还原后再进行分离. 这是由于含硝基不对称
酞菁混合物之间的极性相近，而将硝基还原成氨基后， 虽然柱层析分离时会有些拖尾，但它们的极性相差比较 大，从而大大降低分离的难度.

\section{4 含对叔丁基苯氧基的对称及不对称型酞菁的紫外 可见光谱分析}

以二氯甲烷为溶剂，分别测定了对称型酞菁 9 和不 对称酞菁 $12,13,14$ 等 4 种配合物的紫外可见光谱，如图 1 所示. 这些配合物在紫外可见光区都有两个特征吸收 带，即在 $680 \mathrm{~nm}$ 附近可见光区的 $\mathrm{Q}$ 带以及在 $340 \mathrm{~nm}$ 附 近的近紫外区的 B 带 ${ }^{[22]}$, 而且它们的吸收峰位基本没有 发生变化，这是因为这些配合物的主要取代基和中心金 属原子是相同的. 但是，不对称酞菁 $12,13,14$ 的 $\mathrm{Q}$ 带吸 收峰形状和光谱宽度均不同于对称型酞菁 $\mathbf{9}$, 且光谱吸 收范围更宽. 因此, 不对称酞菁可能会有着更好的光学 性能.

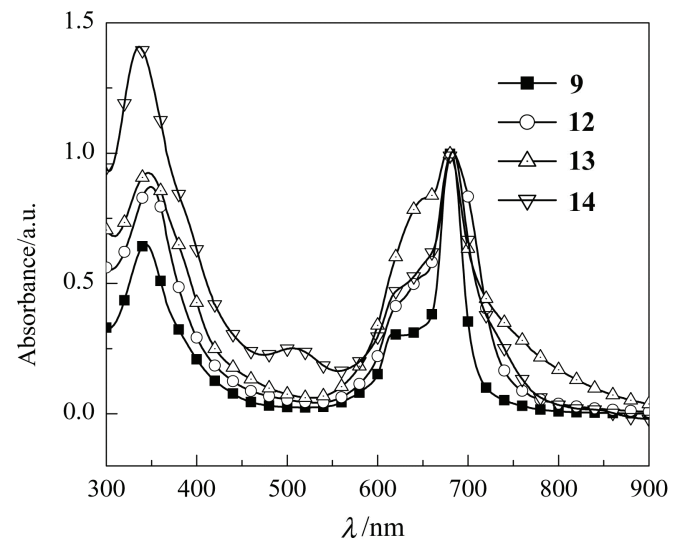

图 1 化合物 9, 12, 13, 14 的紫外可见吸收光谱 $\left(10^{-4} \mathrm{~mol} / \mathrm{L}\right)$ Figure 1 UV-vis absorption spectra of compounds 9, 12, 13 and $14\left(10^{-4} \mathrm{~mol} / \mathrm{L}\right)$

\section{3 小结}

通过对一系列对称型酞菁 $\left(\mathrm{A}_{4}\right)$ 的合成及溶解性研 究，发现取代基的空间位阻越大，酞菁的聚集体就越难 形成, 因而酞菁的溶解性越好. 因此, 我们采用 4-对叔 丁基苯氧基邻苯二甲腈为前躯体，合成了含有氨基和羧 基的不对称酞菁 $\left(\mathrm{A}_{3} \mathrm{~B}\right)$, 并对合成路线进行了优化. 另 外, 不对称酞菁 12 和 14 分别带有一个氨基和羧基, 还 可以进一步修饰(例如与核酸、多肽以及其它功能单元 进行共价偶联), 可能成为重要的中间体分子.

\section{References}

[1] Mack, J.; Kobayashi, N. Chem. Rev. 2011, 2, 281.

[2] Claessens, C. G.; Blau, W. J.; Cook, M.; Hanack, M.; Nolte, R. J. M.; Torres, T.; Wohrle, D. Monatsh. Chem. 2001, 132(1), 3.

[3] Zhao, H.-L.; Yao, K.-S. Chem. Ind. Eng. Prog. 2008, 27, 1887 (in Chinese). 
(赵海丽，姚开胜，化工进展, 2008, 27, 1887.)

[4] Wang, B.-R. Organic Syntheses and Reactions, Science Press, Beijing, 1985, pp. 810 817 (in Chinese). (王葆仁, 有机合成反应, 科学出版社, 北京, 1985, pp. 810 817.)

[5] Xie, W.-W.; Xu, H.-T.; Gan, C.-S.; Pan, Z.-X.; Yan, T.-T.; Peng, B.-X. Chin. J. Chem. Phys. 2003, 16, 491.

[6] Gao, J. S.; Cheng, C. W.; Zhou, X. C.; Li, Y. Y.; Xu, X. Q.; Du, X. G.; Zhang, H. Q. J. Colloid Interface Sci. 2010, 342, 225.

[7] Baugh, S. D. P.; Yang, Z. W.; Leung, D. K.; Wilson, D. M.; Breslow, R. J. Am. Chem. Soc. 2001, 123, 12488.

[8] Brewis, M.; Clarkson, G. J.; Humberstone, P.; Makhseed, S.; McKeown, N. B. Chem. Eur. J. 1998, 4, 1633.

[9] Tararykina, T. V.; Maizlish, V. E.; Galanin, N. E.; Shaposhnikov, G. P.; Bykova, V. V.; Usol'tseva, N. V. Russ. J. Org. Chem. 2007, 43, 1719.

[10] Zhang, X.-F.; Xu, H.-J. Chem. J. Chin. Univ. 1994, 15, 917 (in Chinese).

(张先付, 许慧君, 高等学校化学学报, 1994, 15, 917.)

[11] Leznoff, C. C.; Hu, M.; Nolan, J. M. Chem. Commun. 1996, 1245.

[12] Tomoda, H.; Saito, S.; Ogawa, S.; Shiraishi, S. Chem. Lett. 1980, 1277.

[13] Kliesch, H.; Weitemeyer, A.; Muller, S.; Wohrle, D. Liebigs Ann.
1995, 1269

[14] Qiu, L.; Shen, Y.-J.; Xu, H.-J.; Yuan, P.; Xia, Z.-J.; Zou, Y.-H. Acta Chim. Sinica 1997, 55, 37 (in Chinese).

(邱玲, 沈玉全, 许慧君, 袁平, 夏宗矩, 邹英华, 化学学报, 1997, 55, 37.)

[15] Nie, J.-T.; Duan, W.-B.; Yang, S.-L.; Chen, N.-S. Chin. J. Inorg. Chem. 1996, 9, 246 (in Chinese).

(聂静涛，段武彪，杨素苓，陈耐生，无机化学学报，1996，9， 246.)

[16] Hu, M. G.; Brasseur, N.; Yildiz, S. Z.; Lier, J. E.; Leznoff, C. C. J. Med. Chem. 1998, 41, 1789.

[17] Tomoda, H.; Saito, S.; Shinsaku, S. Chem. Lett. 1983, 313.

[18] Sibrian-Vazquez, M.; Ortiz, J.; Nesterova, I. V.; Femandez-Lazaro, F.; Sastre-Santos, A.; Soper, S. A.; Vicente, M. G. H. Bioconjugate Chem. 2007, 18, 410.

[19] Alzeer, J.; Vummidi, B. R.; Roth, P. J. C.; Luedtke, N. W. Angew. Chem., Int. Ed. 2009, 48, 9362.

[20] Kudrevich, S. V.; Ali, H.; Vanliter, J. E. J. Chem. Soc. 1994, 2767.

[21] Mori, S.; Nagata, M.; Nakahata, Y.; Yasuta, K.; Goto, R.; Kimura, M.; Taya, M. J. Am. Chem. Soc. 2010, 132, 4054.

[22] Walter, M. G.; Rudine, A. B.; Wamser, C. C. J. Porphyrins Phthalocyanines 2010, 14, 759. 